\title{
O lugar da cultura no contexto pós-Onze de Setembro
}

The Place of Culture After September 11, 2001

Le lieu de la culture dans le contexte du post-11 septembre

\section{George Yudice}

\section{OpenEdition}

\section{Journals}

Edição electrónica

URL: http://journals.openedition.org/rccs/1273

DOI: $10.4000 /$ rccs. 1273

ISSN: 2182-7435

\section{Editora}

Centro de Estudos Sociais da Universidade de Coimbra

Edição impressa

Data de publição: 1 Outubro 2002

Paginação: 175-187

ISSN: 0254-1106

\section{Refêrencia eletrónica}

George Yudice, "O lugar da cultura no contexto pós-Onze de Setembro », Revista Crítica de Ciências Sociais [Online], 63 | 2002, colocado online no dia 01 outubro 2012, criado a 19 abril 2019. URL : http:// journals.openedition.org/rccs/1273; DOI : 10.4000/rccs.1273 


\section{GEORGE YUDICE}

New York University

\section{O lugar da cultura no contexto pós-Onze de Setembro}

Este ensaio discute os efeitos que os novos sistemas de segurança e vigilância postos em prática após os ataques do 11 de Setembro têm sobre a cultura. Já transformada pelos processos da globalização e, efectivamente, contribuindo para esses processos, a cultura constitui um espaço primordial de conflito e controlo na sequência dos ataques: movimentos contestatários, muitos dos quais são culturalmente constituídos, são identificados com o terrorismo; os filmes de Hollywood, a imprensa electrónica e a imprensa tradicional são recrutados ao serviço da segurança; planeiam-se novas formas culturais de controlo. Em última análise, a guerra ao terrorismo também consegue proteger o regime de acumulação estabelecido pelo Consenso de Washington. Será o movimento anti-globalização capaz de enfrentar o novo regime de acumulação?

A tese que proponho neste ensaio é que as consequências dos atentados do onze de Setembro, sobretudo o fortalecimento dos aparelhos e tecnologias de segurança e vigilância não só nos EUA mas em todo o mundo, abrem uma janela interessante para essa turbulência que Boaventura de Sousa Santos detecta na coexistência de interpretações paradigmáticas e subparadigmáticas com respeito à transição entre a actual hegemonia do capitalismo neoliberal e um novo modo de acumulação, já apontado pelo pós-consenso de Washington, que ele menciona, citando Stiglitz (Santos, 2001: 88). É muito cedo ainda para adivinhar se essas consequências darão maior apoio a uma ou outra interpretação, mas é evidente que as acções dos EUA procuram estabelecer uma ordem onde parece haver grande desordem. $\mathrm{Na}$ encruzilhada actual, desordem e ordem se alimentam reciprocamente, pois as intervenções dos Estados Unidos no Médio Oriente e na Ásia do Sul têm desencadeado uma longa série de blowbacks ou ricochetes que, ao mesmo tempo que aumentam a turbulência, também legitimam o militarismo e as agências de segurança.

Um segundo aspecto da minha proposta é que essa tentativa de ordem não é só militar mas também profundamente cultural. Tal deve-se, em pri- 
meiro lugar, a que o capitalismo neoliberal, ao procurar debilitar o Estado-Providência (seja do tipo que for) por meio das instituições financeiras internacionais e multilaterais que impõem ajustamentos estruturais, desencadeia uma miríade de recomposições de identidades estabelecidas em relação à malha institucional de um país particular. Essas recomposições operam sobre as necessidades ligadas às identidades que, segundo Nancy Fraser (1989), se abrem à interpretabilidade derivada da luta de interesses que circulam e colidem nas instituições do Welfare State. Trata-se de uma renegociação dos termos da cidadania, que se culturaliza na medida em que se presta à interpretabilidade das necessidades e dos direitos estabelecidos. É evidente que a definição de operário, grupo étnico, minoria, imigrante, família, sexualidade, etc., assim como as necessidades atribuídas a essas identidades, mudam de acordo com esse processo e com a transição para uma outra ordem social.

Cabe assinalar, além disso, que o cálculo de risco que representam essas identidades para os poderes sociais, políticos e económicos também muda de um regime para outro. $\mathrm{Na}$ actual conjuntura, não existem condições para negociar um compromisso keynesiano, por todas as razões que em geral se agrupam nas teorias da sociedade pós-industrial, pós-fordista, pósou transnacional: flexibilização produtiva e laboral, privatização, desregulação, tecnificação, financeirização, terceirização, ou surgimento do sector privado não lucrativo, etc. Esse "novo quadro legal adequado à liberalização dos mercados, dos investimentos e do sistema financeiro," sobre o qual escreve Boaventura de Sousa Santos (2001: 49), também implica novos conceitos de risco. Os imigrantes apresentam sempre uma ambiguidade em relação ao risco: por um lado, são necessários para proporcionar mão-de-obra barata, mas, por outro, são considerados uma fonte de inquietação, sobretudo no que poderíamos chamar a sociedade pós-disciplinar, onde a proliferação de identidades culturais já não é governada pela escola, a igreja, o clube social, etc. A sociedade disciplinar coincide com a modernidade e com a legitimidade para as autoridades que geram os critérios de valor. Numa sociedade pós-moderna, não impera uma única escala de valores e a legitimidade dos saberes institucionalizados é questionada por múltiplos actores, cada um com escalas ou normas diferentes. Como veremos mais abaixo, surge um novo princípio de autoridade num contexto pós-disciplinar: a sociedade de controlo.

É justamente a falta de uma sociedade disciplinada, de uma sociedade civil unificada, que Robert Putnam (2000) lamenta na actualidade, pois, segundo ele, essa unidade é fundamental para o bem-estar social. Para Samuel Huntington (1996), como para Chrisopher Lasch (1978) vinte e 
cinco anos atrás, são precisamente as práticas do culto do self e a formação de subgrupos identitários, que Graça Carapinheiro descreve na sua contribuição para Globalização: Fatalidade ou utopia?, que geraram essa fragmentação e falta de coesão que enfraquece a sociedade estado-unidense e a abre a ataques externos, tanto civilizacionais como terroristas. Putnam conjectura que o trauma do onze de Setembro deveria produzir uma coesão civil - serviços multi-religiosos, ajuda cidadã, serviços cívicos como os dos boy scouts, etc. - semelhante à que se deu depois do ataque a Pearl Harbor em 1941. Mas não diz nada a respeito da crítica que pode fazer-se ao neoliberalismo nem a essa sociedade civil que se compatibiliza com ele.

Para Huntington, a tolerância estado-unidense relativamente à diversidade cultural enfraquece o carácter moral da nação, levando ao risco de colapso da civilização ocidental. É interessante que o governo de Bush não tenha seguido o raciocínio de Huntington e tenha recusado os alardes de superioridade ocidental - ao contrário de Berlusconi - e promovido a aliança com as nações islâmicas para combater o terrorismo. O presidente dos EUA declarou repetidamente que, dentro dos EUA, os muçulmanos estado-unidenses são patrióticos cidadãos americanos. Bush aproveitou a retórica da diversidade multicultural para criar uma ponte entre a agenda nacional de unidade e a agenda política de conseguir o apoio internacional para os ataques militares contra países que, segundo os serviços de vigilância, abrigam terroristas. Pior ainda, segundo Bush, esses mesmos países reprimem a diversidade.

É evidente que, no contexto estado-unidense, o multiculturalismo perdeu o seu gume crítico e tornou-se mais num instrumento de consenso, poucos anos depois das guerras culturais, quando se acreditava que a sociedade se decompunha num conjunto de tribos heterogéneas. O governo chegou mesmo a celebrar a tolerância em relação à diversidade como característica exclusiva da sociedade estado-unidense. Quase nunca referidos antes como americanos "hifenizados", começou-se a ouvir falar em Arab-Americans e Pakistani-Americans. O hífen funciona como recurso cultural para compatibilizar o Islão com o credo americano do "E pluribus unum". Por exemplo, os jornais reportaram que um grupo de Pakistani-Americans foi ao seu país de origem como embaixadores de boa vontade (good will ambassadors) para explicar por que os EUA são um país que deve ser amado (Domowitz, 2001).

Mas o outro lado da ambiguidade relativamente aos imigrantes expressou-se com igual intensidade, embora de modo menos espectacular. Ao mesmo tempo que o governo celebrava os Arab-Americans e muçulmanos e repudiava os ataques contra eles, também iniciou uma caça dis- 
criminatória contra eles. Até um guarda-costas de Bush, um Arab-American, foi detido num aeroporto ("Guard for Bush", 2001). O mesmo Bush que celebrou a diversidade - seu gabinete ministerial é o mais etnicamente diverso na história dos EUA - autorizou, mediante o USA Patriot Act, julgamentos militares a milhares de pessoas, quase todas só por terem aspecto suspeito, quer dizer, árabe. Mais de 5.000 apresentaram-se "voluntariamente", segundo comunicado do Departamento de Estado, e desses, 1200 ficaram indefinidamente na cadeia, sem serem culpados de cometer crimes nem de transgredir as leis de imigração (Nojeim, 2001; ACLU, 2002).

Estas medidas correspondem aos "sistemas de vigilância e de governo para produzir controlo e regulação" (Carapinheiro, 2001: 202). Como veremos, esses sistemas para conter o risco (ou a percepção do risco, que, no campo económico, talvez seja a mesma coisa) podem formar parte de estratégias secretas ou espectaculares. Nos primeiros dias após os atentados do onze de Setembro, a população estado-unidense, ante a incessante repetição mediática do colapso das torres, queria ver manifestações de controlo. Esses atentados pareciam um pastiche dos filmes hollywoodianos de acção, mas faltavam os heróis - Stallone, Schwarzenegger ou Willis - para combaterem os terroristas. Faltava uma manifestação de força oriunda da Casa Branca; em vez disso, o Presidente Bush encorajou a população a sair às compras e viajar em aviões, tanto para mostrar aos terroristas que um americano não tem medo quanto para combater o risco económico devido à queda no consumo.

Parecia que Bush e seu governo esperavam que esta performance - que é um fenómeno fundamentalmente cultural - serviria para sarar a enorme ferida que se sofreu tanto na perda de vidas humanas e de propriedade, quanto no simbólico. Esses dois aspectos são inseparáveis na performance económica. Como explica o relatório do FMI, World Economic Outlook: The Global Economy after September 11, os desastres produzem uma queda na confiança pública relativamente ao futuro que se traduz numa queda do consumo que, por sua vez, produz uma queda na demanda de mercadorias e aumenta os custos de segurança (International Monetary Fund, 2001). O relatório fala em muitas outras consequências, mas uma que vale a pena mencionar é a queda no turismo, que é fatal para as cidades (embora o turismo nos lugares considerados seguros, como Portugal, possa aumentar). E com a queda no turismo, por sua vez, dá-se um efeito muito negativo na produção de cultura profissional: teatro, concertos, museus, galerias, etc.

São necessários sistemas de segurança para o sector cultural, pois ele opera como um dos recursos mais importantes da acumulação, sobretudo no que respeita ao fomento à inovação de que falam Castells e Rifkin. 
Segundo estes autores, são as ideias que têm maior valor na nova economia. Portanto, é essencial compreender que tipos de cultura são incentivados e quais são desincentivados pelas consequências do onze de Setembro. O novo regime de vigilância e segurança ameaça os movimentos sociais e outras iniciativas contestatárias, neutralizando-os repressivamente e absorvendo-os nos mecanismos de segurança, que adoptam várias formas sociais e culturais. Daí a importância das políticas culturais neste novo período. A globalização gerada pela transnacionalização das empresas e os fluxos financeiros tem produzido maior desigualdade do que nos vinte e cinco anos depois da Segunda Guerra Mundial. Mas, em vez de desenhar políticas para contrariar a pobreza gerada pela globalização, as instituições financeiras e comerciais internacionais (FMI, Banco Mundial, OMC, etc.) limitam-se a criar novos programas para reduzir ou eliminar a enorme dívida externa dos países mais pobres, ao mesmo tempo que recorrem cada vez mais à cultura como panaceia (ver as declarações de Wolfensohn, 2000). Mas mesmo que se elimine a dívida externa dos países pobres, isso não diz respeito à estrutura que gera a desigualdade, que é a juridificação da divisão internacional do trabalho e a produção de propriedade.

A transformação dos sistemas de comunicação mediante processos neoliberais (privatização, desregulação, eliminação dos serviços proporcionados pelo Estado-Providência por razões políticas e económicas) resulta numa recomposição e ressignificação de territórios e públicos. A transnacionalização e (neo)liberalização das indústrias culturais impõe (1) a necessidade de inserção numa economia supranacional e (2) reestruturações para facilitar essa inserção que respondem a "uma dialéctica de uniformização e de diferenciação” (Herscovici, 1999: 58). Por um lado, uniformizam-se protocolos jurídicos, tecnologias, formas administrativas; por outro, a acomodação ao mercado transnacional requer a geração de diferenças locais que tornem possível o lucro dos conteúdos além das fronteiras territoriais. "[C]ada espaço geográfico precisa de se diferenciar e de construir a sua imagem mediática com o objectivo de se valorizar em relação ao exterior para se inserir nessas redes internacionais; a cultura é amplamente utilizada na construção dessa imagem mediática" (Herscovici, 1999: 58-59). O facto de a valorização de localidades e de seus conteúdos se fazer a partir da geração dessas diferenças (orquestradas desde logo pelo contexto do mercado transnacional) obriga os processos de formação de identidades culturais a obedecerem a mandatos performativos. Noutras palavras, as diferenças não podem ser pensadas fora do contexto em que derivam seu valor; daí que se argumente que as diferenças se constituem dentro dos processos de globalização (Lacarrieu, 2000: 4-5). 
Tomar consciência disso complica a nossa compreensão dos programas de reivindicação do local, pois a diferença é o recurso que permite a valorização, inclusive de iniciativas da sociedade civil que não correspondem directamente ao mercado. Os territórios que não podem responder ou não respondam ao imperativo performativo projectado a partir do campo de forças serão excluídos ou marginalizados nos processos de produção de valor. A culturalização económica só aumenta a subordinação dos subalternos, ao mesmo tempo que põe em circulação um discurso sobre a diferença que pareceria incluí-los.

Um dos passos mais importantes desta culturalização da economia ou economização da cultura é a subsunção do trabalho intelectual, imaterial e afectivo ao processo de produção e acumulação. Essa subsunção, facilitada pelas novas tecnologias informacionais e comunicacionais, toma três formas. Primeiro, a informatização e concomitante transformação do processo de produção, com o resultado de que até a manufactura se torna um serviço. Segundo, a manipulação de símbolos, que se bifurca, por um lado, no trabalho criativo e analítico e, por outro, no trabalho rotineiro, como na operação da caixa registadora no hipermercado. Em terceiro lugar, o investimento do afecto na economia de serviços como os de saúde, entretenimento e aquilo a que Castells (2000) chama "dar vida" (bares, catering, etc.) e Rifkin (2000) “compra e venda de experiências”. Esta terceira forma de trabalho, menos dependente da alta tecnologia do que as outras formas, é também uma das fontes mais importantes, segundo Castells e Volkerling (2001), do que satisfaz os trabalhadores intelectuais e criativos que se concentram nos lugares de inovação que impulsam a nova economia. Por sua vez, este novo regime de acumulação requer uma estrutura legislativa e jurídica internacional que facilite a geração de valor a partir do imaterial, que é facilmente expropriável e reproduzível, por exemplo, com a mesma tecnologia de informação, e do serviço afectivo, que é facilmente explorável.

$\mathrm{Na}$ minha opinião, os mecanismos legislativos e jurídicos que criam e sustentam os direitos de propriedade intelectual constituem o eixo que possibilita o funcionamento dessa complexa engrenagem do novo regime de acumulação. São os transformadores, por assim dizer, do património - material ou imaterial - em propriedade. O lucro deriva da posse dos direitos (ou, como diria Storper (1989), da criação de propriedade onde antes não havia ou onde era património da humanidade, para ecoar um dos termos que emprega Boaventura de Sousa Santos). Os que não têm esses direitos ou os perderam devido à legislação favorável às empresas ou são trabalhadores contratados que fornecem serviços e conteúdos. Porque a acumulação se baseia na geração de propriedade intelectual, os países e as cidades 
mais conscientes desta fonte de acumulação têm criado políticas integrais para fomentar a criação de propriedade intelectual em biotecnologia, informática e em "conteúdo cultural". Assim, asseguram que as empresas sediadas no seu território continuem a dominar o comércio de propriedade intelectual. Michael Volkerling (2001) analisa as políticas para fomentar "economias criativas" no Reino Unido, Canadá, Austrália, Nova Zelândia e alguns países da União Europeia. Eu mesmo tenho pesquisado as políticas dos EUA a este respeito, como, por exemplo, as medidas tomadas pela Intellectual Property and the National Information Infrastructure of the White House Information Infrastructure Task Force (IITF), que incluem o reforço dos regimes de copyright para assegurar a competitividade dos Estados Unidos na nova economia. Segundo o relatório deste comité, "por mais que se multipliquem os computadores, telefones, scanners, impressoras, switches, routers, cabos, redes e satélites não se logrará criar uma bem sucedida infra-estrutura nacional de informação (NII) se não houver conteúdo. O que impulsará o sucesso é o conteúdo que circular pela infra-estrutura informacional nacional (NII)" (United States, 1995).

É por essa razão que os EUA consideram o ciberterrorismo uma das formas mais perigosas de ataque. A protecção da infra-estrutura informacional dos EUA é um dos itens incluídos no orçamento para a segurança nacional, que alcançará 38 mil milhões de dólares neste ano, o dobro do ano passado (Stevenson, 2002). Os recursos para emergências aumentaram mais de $1.000 \%$, de 291 milhões de dólares para 3 mil milhões e meio, e as tecnologias informáticas de segurança aumentaram em 700 milhões de dólares(Miller, 2002). Ao mesmo tempo que se aumenta o orçamento de segurança contra o terrorismo, o sistema de segurança social sofre reduções: a protecção laboral sofrerá uma queda de $7 \%$; a protecção ambiental uma queda de 3,6\%; e os subsídios agrícolas e a educação não terão aumento. Como já observaram vários comentaristas, a segurança nacional é boa para o sector económico e ajudará a tirar aos EUA da recessão. Sobretudo, essa política é óptima para as empresas que se especializam em cibersegurança. Trata-se de uma extensão da protecção para os negócios, cujas operações passam quase totalmente por via electrónica.

Também se trata da protecção dos lugares de inovação e da infra-estrutura cultural que, como indiquei antes, a reproduz. Por exemplo, é uma prática bastante normalizada montar câmaras de circuito fechado (CCTV) em cidades com uma nova infra-estrutura cultural. É o caso de Bilbau, que hoje em dia é considerada o modelo para o desenvolvimento urbano através da cultura. Preocupados com a deterioração da sua infra-estrutura industrial e a má reputação terrorista, os dirigentes políticos e empresariais procuraram 
revitalizar Bilbau investindo numa infra-estrutura cultural que atraísse turistas e constituísse a base de um novo complexo industrial de serviços, informação e cultura. A “compra” de um museu Guggenheim desenhado por Frank Gehry proporcionou o íman para atrair essas actividades que "dão vida": "junto ao trabalho de inovação desenvolve-se o tecido social de bares, restaurantes, encontros na rua, etc., que dão vida a esse lugar" (Castells, 2000). ${ }^{1}$ "O conhecimento, a cultura, a arte [...] ajudarão a lançar Bilbau nessa breve lista de capitais mundiais", segundo Alfonso Martínez Cearra, director de Bilbao Metrópoli 30, uma rede de funcionários governamentais, empresários, educadores, directores de ONGs e executivos dos meios que lideram o desenvolvimento da cidade (Jacobs, 1997: 14).

Mas essas iniciativas também têm desvantagens, como no caso do Pelourinho, a praça central do bairro Maciel em Salvador da Bahia, e o lugar de maior simbolismo afro-brasileiro, pois os escravos eram ali punidos e vendidos em leilão. Uma rede composta por grupos culturais (incluindo a banda Olodum), empresas, fundações, ONGs, instituições do governo municipal e federal, bancos, a igreja e a indústria do turismo realizou este projecto que desalojou os residentes pobres, de maneira que, embora seja o cenário da cultura afro-bahiana para os turistas, os que vivem essa cultura já não têm lugar ali (Gomes, 1999: 13). Vemos com clareza como um bem patrimonial, quer dizer público, é transformado em bem privado para gerar lucro.

A privatização requer vigilância porque é necessário criar um ambiente "seguro" para os turistas ou a classe profissional-executiva. No metro de Bilbau, por exemplo, foram instaladas câmaras de vigilância em cada estação para controlar as actividades dos passageiros (Jacobs, 13-14). Essa vigilância assumiu uma forma ainda mais intrusiva em Peekskill, cidade pós-industrial a uma hora de Nova Iorque, que uma década atrás atraiu artistas deslocados de Soho com incentivos como últimos andares baratos. Em pouco tempo, Peekskill foi "descoberta” pelos que David Brooks designa como "doidos do paraíso", que "tomam capuccinos gelados em bares da moda" (apud Oropeza, 2001). O reverso desse "paraíso" são as câmaras instaladas em cada esquina sob o alibi de que era necessário controlar o narcotráfico. Mas os antigos residentes, muitos dos quais são minorias raciais e estão desempregados devido à fuga da indústria para as maquiladoras do terceiro mundo, protestam que a "vida cultural" oferecida aos profissionais

\footnotetext{
${ }^{1}$ Castells acrescenta a respeito do caso que comenta: "Neste momento, [o sector cultural] está a passar a ser, depois da indústria financeira, a segunda indústria mais dinâmica da cidade de San Francisco".
} 
lhes é vedada (Peterson, 1999). É evidente que se aproveita o discurso e a prática da segurança para satisfazer a compulsão de culpar os estrangeiros e os excluídos sociais pelos perigos que se sentem nesta conjuntura de grande instabilidade.

Os atentados do onze de Setembro deram maior legitimação para impor políticas de vigilância e segurança. Além dessas políticas mais simples de vigilância em CCTV, também se impõem novas tecnologias com potencial para invadir todo o espaço do sujeito. Por exemplo, os novos aparelhos biométricos para reconhecimento ocular e os scanners retinais, que obrigam as pessoas a mostrar a cara para o scanning electrónico (Kilgannon, 2002). Outra nova tecnologia são os documentos inteligentes de identificação (smart cards), que contêm nas suas fitas magnéticas informação que poderá ser usada pela polícia e outros serviços de segurança. Estas tecnologias, por sua vez, requerem enormes bases de dados para a detecção e reconhecimento de criminosos e pessoas perigosas. Mas, como explica David Lyon (2001), especialista em cibersegurança, essas tecnologias terão provavelmente consequências não premeditadas, como o fortalecimento de práticas informais de divisão e exclusão social.

E também servem para combater os movimentos que se organizam para criticar e transformar as estruturas económicas, políticas e culturais que geram tanta desigualdade. Os novos sistemas de segurança procuram parecer-se cada vez mais com as estruturas reticulares flexíveis de terroristas e activistas democratizantes. Como explicam John Arquilla e David Ronfeldt - assessores do Instituto Nacional de Pesquisa em Defesa, um centro de pesquisa e desenvolvimento financiado pelo Gabinete do Secretário da Defesa, os Chefes do Estado Maior e outras agências de defesa - a única forma de combater uma rede é organizar-se em rede e atacar de surpresa em grupos que são flexíveis e têm capacidade para actuar imediatamente. A eficácia provém não da acção em massa, mas da constante dispersão e da forma de acondicionamento (packeting). Por exemplo, os narcotraficantes podem dividir grandes carregamentos de drogas em pequenos pacotes para o transporte simultâneo através de várias fronteiras; os activistas de ONGs, como no caso dos Zapatistas, têm bastante diversidade nos seus quadros para responderem a qualquer acontecimento - por exemplo, violação de direitos humanos ou ambientais (Arquilla e Ronfeldt, 2001: 13). Outros exemplos que os autores apresentam são a resistência tchetchena e a batalha anti-globalização de Seattle.

As estratégias de vigilância e segurança chegam ao mais essencial da cultura: a transformação da informação em inteligência e em narração. Segundo Arquilla e Ronfeldt, os que têm melhor inteligência ganharão a guerra das 
redes humanas. Entre as suas recomendações para combaterem os backers, eles incluem: infiltrar as redes inimigas e fazer acreditar ao mais tonto que ele é o mais importante do núcleo; discernir as figuras mais perigosas e questionar a sua lealdade ao grupo; controlar as histórias que as pessoas contam quando se explicam e actuam. Segundo os autores, a narrativa terrorista "dá aos seus seguidores uma causa comum - um nós contra eles. Pareceria que neste momento os EUA têm vantagem a este respeito à escala mundial, mas também vemos os palestinianos dançando na rua [festejando o colapso das torres gémeas]. Uma parte daquela narrativa é que a América é malévola e que a presença da América é a causa de tantos dos problemas do Médio Oriente. Nós temos que atacar essa parte da narrativa" (Arquilla e Ronfeldt, 2001).

Essa engenharia narrativa consegue-se desprestigiando o inimigo; destruindo as suas redes de apoio; infiltrando-o com valores diferentes, portanto, dividindo-o e conquistando-o; aumentando o número de contra-redes humanas na própria sociedade civil. Quanto aos espectáculos, opina-se que os ataques militares já não constituem a única acção efectiva, pois cada vez que erram com os seus mísseis, mostram que não têm informação fiável e, além disso, tornam possível construir narrativas contra nós (Karen Stephenson, apud Garreau, 2001).

Para concluir, considero duas opções a respeito do lugar da cultura na nova encruzilhada. A primeira é aquela que os militares tomaram ao convidar cineastas e argumentistas como Steven E. De Souza e Joseph Zito, realizadores de Delta Force One, para gerar novos cenários de terrorismo mediático (Roberts, 2001). Também se usam as novas tecnologias de simulação de Hollywood para treinar os militares (Bart, 2001; Hart, 2001; "U.S. Army Goes Hollywood”, 1999). Este uso dos media aponta, sem dúvida, para um tipo de estudo muito diferente dos que se fazem nos departamentos de comunicações. Uma das percepções derivadas desta nova economia política e cultural dos media é que quanto mais os utilizadores participam nos cenários pré-escritos para interpretar o mundo, tanto mais controlo se consegue para o novo regime de vigilância e segurança cultural. Pelo menos, essa é a premissa dos ciber-terrorólogos. Poderão estar errados.

Tudo o que estou a apresentar aqui acomoda-se à ideia da sociedade de controlo, que não consiste só nessas tecnologias de segurança mas também no incentivo a associações reticulares e a ONGs que disseminam padrões de comportamento e valores, aquilo a que Foucault chamou governamentalidade. Com a diminuição das instituições estatais disciplinadoras, incumbe às associações reticulares produzirem vida; elas também instrumentalizam o biopoder e, analogamente, o poder cultural. Por exemplo, as ONGs que 
operam no Sul não só fomentam a democratização mas nesse mesmo processo enxertam práticas de auto-estima e conceitos de cidadania segundo padrões internacionais. A sociedade de controlo não é aquela em que a disciplina só se dissemina a partir de instituições estatais - os aparelhos ideológicos estatais de Althusser - mas aquela em que o biopoder se difunde capilarmente em toda a sociedade.

Como vimos, pelo menos no caso dos EUA, o Estado está longe de se retirar; é ele que gera vigilância e segurança, embora seja mais para proteger o capital do que os cidadãos. As suas políticas de propriedade intelectual - até nos casos de bioterrorismo, quando outras nações menosprezaram os direitos das empresas farmacêuticas para produzirem antídotos genéricos - restringem as possibilidades de autoconstituição individual, para ecoar palavras de Carapinheiro (2001: 225).

$\mathrm{Na}$ minha opinião, o movimento antiglobalização tem que estender a sua acção além das críticas à exploração da natureza e dos operários em sweatshops e maquiladoras. Tem-se gerado também uma maquilização da produção e da experiência cultural. A recente vitória (ou quase vitória) do Brasil, África do Sul e Índia na OMC a respeito da isenção dos direitos de propriedade intelectual em casos de crise de saúde pública (como é o caso da SIDA) inspira optimismo, embora ainda não tenham mudado os princípios fundamentais nem os acordos internacionais. Mas, mesmo assim, tem que aplicar-se esse tipo de luta aos direitos de propriedade intelectual no que respeita à cultura. Para isso, incumbe-nos a nós compreendermos melhor como o capital se apropria do valor da cultura, a experiência mesma, como essa expropriação é protegida pelo regime de acumulação e como pode ser combatida.

\section{Referências Bibliográficas}

ACLU (2002), «Safe and Free in Times of Crisis», comunicado de imprensa. Disponível em: $<$ http://www.aclu.org/ safeandfree/index.html $>$.

Arquilla, John; Ronfeldt, David (orgs.) (2001), Networks and Netwars: The Future of Terror, Crime, and Militancy. Santa Monica. Rand. Disponível em: <http:// www.rand.org/publications/MR/MR1382/>.

Bart, Peter (2001), «Bush Crusade Goes Hollywood: Will He Bomb at Box Office?», Variety, 7/11. Disponível em: <http://dailynews.yahoo.com/htx/nm/20011107/en/ industry-war_1.html>.

Carapinheiro, Graça (2001), «A globalização do risco social», in B. S. Santos (org.), Globalização: Fatalidade ou utopia?. Porto: Afrontamento, 197-229. 
Castells, Manuel (2000), «La ciudad de la nueva economía», La factoría, 12 (Julho-Agosto). Disponível em: <http://www.lafactoriaweb.com/articulos/castells12.htm>.

Domowitz, Susan (2001), «Pakistani-Americans Talk to Pakistanis about Muslim Life in the U.S», U.S. State Department, 19/11. Disponível em: <http://usinfo.state.gov/ topical/pol/terror/01111915.htm>.

Fraser, Nancy (1989) «Women, Welfare, and the Politics of Need Interpretation», in N. Fraser, Unruly Practices: Power, Discourse and Gender in Contemporary Social Theory. Minneapolis: University of Minnesota Press, 144-160.

Garreau, Joel (2001), «Disconnect the Dots», 17/11. Disponível em: <http:// www.washtech.com/news/regulation/12516-1.html>.

Gomes, Marco A. de Filgueiras (1999), Au coeur de Babia: Le Pelourinho, histoire et défis contemporains. Paris: Tout pour Plaire Multimédia.

"Guard for Bush Isn't Allowed Aboard Flight." (2001), The New York Times, 27/11. Hart, Hugh (2001), «Bringing Hollywood Pizazz to Military Training», The New York Times, 15/11.

Herscovici, Alain (1999), «Globalización, sistema de redes y estructuración del espacio: un análisis económico», in Guillermo Mastrini; César Bolaño (orgs.), Globalización y monopolios en la comunicación en América Latina. Hacia una economía política de la comunicación. Buenos Aires: Editorial Biblos, 49-60.

Huntington, Samuel P. (1996), The Clash of Civilizations and the Remaking of World Order. New York: Simon and Schuster.

International Monetary Fund (2001), World Economic Outlook: The Global Economy after September 11. Washington, D.C.: International Monetary Fund. Disponível em: <http://www.imf.org>.

Jacobs, Karrie (1997), «Capital Improvements», Guggenheim Magazine, Fall, 10-17. Kilgannon, Corey (2002), «Cameras to Seek Faces of Terror in Visitors to the Statue of Liberty», The New York Times, 25/11.

Lacarrieu, Mónica (2000), «Construcción de imaginarios locales e identidades culturales en la mundialización». Comunicação apresentada ao Seminário Nuevos retos y estrategias de las políticas culturales frente a la globalización, Instituto d'Estudis Catalans, Barcelona, 22-25 de Novembro.

Lasch, Christopher (1978), The Culture of Narcissism: American Life in an Age of Diminishing Expectations. New York: Norton.

Lyon, David (2001), «Terrorism and Surveillance: Security, Freedom, and Justice after September 11 2001». Comunicação apresentada em Open Flows Privacy Lecture Series, 12 de Novembro. Disponível em: <http://privacy. openflows.org/pdf/ lyon_paper.pdf>.

Miller, Judith (2002), «A Nation Challenged: Bioterrorism; Bush to Request a Major Increase in Bioterror Funds», The New York Times, 4/2. 
Nojeim, Gregory T. (2001), «Threats to Civil Liberties Post-September 11: Secrecy, Erosion of Privacy, Danger of Unchecked Government». Comunicado de imprensa. ACLU 14/12. Disponível em: <http://www. aclu.org/news/2001/n121401b.html>.

Oropeza, Mariano (2001), «Un barrio a la carta. Un ensayo sobre estilos de vida y ciudad en un caso», in Ana Wortman (org.), Consumos culturales en una cultura de consumo en la sociedad argentina del ajuste. Buenos Aires: Editorial Del Punto.

Peterson, Iver (1999), «City's Artists Are Its Pride and Something of a Pain», The New York Times, 23/5.

Putnam, Robert D. (2000), Bowling Alone: The Collapse and Revival of American Community. New York: Simon \& Schuster.

Rifkin, Jeremy (2000), The Age of Access: The New Culture of Hypercapitalism, Where All of Life Is a Paid-for Experience. New York: Jeremy P. Tarcher/Putnam.

Roberts, Johnnie L. (2001), «Big Media and the Big Story», Newsweek, 13/10.

Santos, Boaventura de Sousa (org.) (2001), «Os processos da globalização», in B.S.S. (org.), Globalização: Fatalidade ou utopia?. Porto: Afrontamento, 31-106.

Stevenson, Richard W. (2002), «Security and Tax Cuts Win Bush's Protection», The New York Times, 30/1, A27.

Storper, Michael (1989), «The Transition to Flexible Specialisation in the US Film Industry: External Economies, the Division of Labour, and the Crossing of Industrial Divides», Cambridge Journal of Economics, 13, 273-305.

United States. Information Infrastructure Task Force. Working Group on Intellectual Property Rights (1995), Intellectual Property and the National Information Infrastructure: The Report of the Working Group on Intellectual Property Rights/Bruce A. Lehman, Chair. Washington, DC: Office of Legislative and International Affairs, U.S. Patent and Trademark Office, Setembro.

“U.S. Army Goes Hollywood for High-tech Training” (1999). CNN.com, 18/8. Disponível em: <http://www.cnncom/US/9908/18/army.hollywood/>.

Volkerling, Michael (2001), «From Cool Britannia to Hot Nation: 'Creative Industries' Policies in Europe, Canada and New Zealand», The International Journal of Cultural Policy, 7(2).

Wolfensohn, James D. et al. (2000), Culture Counts: Financing, Resources, and the Economics of Culture in Sustainable Development. Washington, D.C.: World Bank. 